\title{
LOCALIZATION OF MULTIPLE TYPES OF JET NOISE SOURCES
}

\author{
Dimitri Papamoschou* and Ali Dadvar ${ }^{\dagger}$ \\ University of California, Irvine, Irvine, California 92697-3975
}

\begin{abstract}
A small-aperture microphone phased array provides noise source maps for turbulent mixing noise emitted from a cold Mach 0.9 jet in two polar directions. The first direction is at 30 degrees to the jet axis and is associated with noise produced by large-scale turbulence. The second direction is at 90 degrees to the jet axis and is associated with noise generated from fine-scale turbulence. The measurements show similarities and differences between the two noise source maps. At given frequency, the axial distributions of large-scale and fine-scale noise sources have comparable shapes. Both types of noise sources peak near the end of the potential core and move towards the nozzle exit as frequency increases. Along the path of peak noise on the space-frequency diagram, the spectra of the two noise sources are distinct and representative of their respective similarity spectra for single point measurement.
\end{abstract}

\section{Nomenclature}

\begin{tabular}{|c|c|c|}
\hline$a$ & $=$ & ambient speed of sound \\
\hline$b$ & $=$ & beam width, $V(x, x \pm b / 2, \omega)=\frac{1}{2} V(x, x, \omega)$ \\
\hline$D$ & $=$ & jet diameter \\
\hline$f$ & $=$ & cyclic frequency \\
\hline$G_{m n}$ & $=$ & cross spectrum matrix \\
\hline$I_{\mathrm{ML}}$ & $=$ & integral of main lobe of point spread function \\
\hline $\mathcal{L}$ & $=$ & axial extent of noise source region \\
\hline$\ell_{m}(x)$ & $=$ & distance of microphone $m$ from focus point $x$ \\
\hline$\ell_{m}(\xi)$ & $=$ & distance of microphone $m$ from point along $\xi$-axis \\
\hline$K$ & $=$ & number of data blocks \\
\hline$M$ & $=$ & number of microphones \\
\hline$N$ & $=$ & number of discrete sources \\
\hline$M_{j}$ & $=$ & jet Mach number \\
\hline$p$ & $=$ & pressure fluctuation \\
\hline$r$ & $=$ & residual \\
\hline$R$ & $=$ & array radius \\
\hline$q(\xi, t)$ & $=$ & noise source strength \\
\hline$s(x, t)$ & $=$ & delay-and-sum array output \\
\hline$S r$ & $=$ & Strouhal number $=f D / U$ \\
\hline$t$ & $=$ & time \\
\hline$U_{j}$ & $=$ & jet velocity \\
\hline$V(x, \xi \omega)$ & $=$ & point spread function $=|W(x, \xi \omega)|^{2}$ \\
\hline$W(x, \xi \omega)$ & $=$ & array pattern \\
\hline$w_{m}$ & $=$ & weight for microphone $m$ \\
\hline $\bar{w}_{m}$ & $=$ & dimensionless weight for microphone $m$ \\
\hline$x$ & $=$ & beam focus point \\
\hline$\epsilon_{m}$ & $=$ & weighted steering vector \\
\hline$\xi$ & $=$ & axial coordinate \\
\hline$\lambda$ & $=$ & wavelength \\
\hline$\Phi(x, \omega)$ & $=$ & array power spectrum $\left(\mathrm{Pa}^{2} / \mathrm{Hz}\right)$ \\
\hline
\end{tabular}

\footnotetext{
*Professor, Associate Fellow AIAA

$\dagger$ Graduate Student Researcher, Member AIAA
} 


$\begin{array}{lll}\Phi_{\mathrm{SPL}}(x, f) & = & \text { lossless array sound pressure level spectrum }(\mathrm{dB} / \mathrm{Hz}) \\ \Psi(x, \omega) & = & \text { distribution of spatially incoherent sources } \\ \tau_{m} & = & \text { time delay for microphone } m \\ \omega & = & \text { radian frequency }=2 \pi f\end{array}$

Subscripts

$\mathrm{a}=$ average among microphones

$m, n=$ microphone indices

$i, k=$ axial indices

wa $=$ weighted average among microphones

\section{Introduction}

Physical understanding and modeling of sound sources in turbulent jets in essential for construction of rigorous predictive models for jet noise emission. For this reason, the jet aeroacoustics community has engaged in a long effort to measure the distribution of noise sources in jets. One of the initial published efforts in the mid-1970s, by Laufer et al., ${ }^{1}$ involved application of an acoustic mirror to a supersonic jet. At the same time, several other groups were working on noise source location using the simultaneous acquisition of signals from two microphones ${ }^{2}$ or multiple microphones. ${ }^{3}$ The theoretical foundation of noise source location using cross-correlations of multiple microphone signals was established by Billingsley and Kinns. ${ }^{3}$ More recently, frequency-domain approaches for processing the microphone array data were introduced by Humphreys et al. ${ }^{4}$ Narayanan et al. ${ }^{5}$ conducted a combined experimental study of the flow characteristics and noise source distribution, using a phased array, of cold and hot subsonic jets. One of the important findings is that the peak noise source is located near the end of the potential core. This study was extended by Lee et al. ${ }^{6}$ who conducted a parametric study of noise source distribution versus jet temperature and Mach number. Hileman et al. ${ }^{7}$ conducted phased array measurements simultaneous with flow visualization to construct instantaneous (time-domain) correlations between the evolution of eddies and far-field sound.

Whether one uses an acoustic mirror or a phased array, the output of the instrument is a convolution between a known kernel (often called the point spread function) and the noise source distribution. In addition, sidelobes caused by spatial aliasing can contaminate the results. Ventatesh et al. ${ }^{8}$ proposed a new beamforming algorithm based on a scheme to minimize contribution of sources outside the focal region. Given that the convolution kernel is known and spurious noise is typically low, an ideal solution would be to deconvolve the array output in order to extract the true noise source distribution. In practice this is a difficult and sensitive operation. Recent studies by Brooks and Humphreys ${ }^{9}$ and by Dougherty ${ }^{10}$ have proposed various methods of deconvolution.

The nature of jet noise sources is very complex and remains a topic of investigation and debate. However, there is wide agreement that, in jets without significant shock cells, there are two distinct types of turbulent mixing noise: noise from large-scale turbulence and noise from fine-scale turbulence. ${ }^{11}$ The former is highly directional and radiates at angles close to the jet axis. The latter is more uniform and affects the sideline. The two noise types have distinct similarity spectra, as established by Tam et al. ${ }^{12}$ In other words, sound emission from a turbulent jet is directive and the nature of the noise sources can be different depending on the polar angle of the observer.

The directional nature of jet noise was in fact captured by the acoustic mirror experiments of Laufer et al. ${ }^{1}$ A recent paper by Tam et al. ${ }^{13}$ provides further introspective into those experiments. However, the known studies on phased-array beamforming applied to turbulent jets have utilized arrays of large aperture that cannot distinguish the aforementioned types of noise. Large aperture is desirable because the spatial resolution (beamwidth) is inversely proportional to the aperture size. The drawback is that one integrates over dissimilar types of jet noise sources.

This paper describes an effort to conduct noise source localization that resolves the two types of turbulent 
mixing noise sources in a subsonic jet. Some theoretical aspects of phased array measurements are introduced, borrowing substantially from the works of Billingsley and Kinns ${ }^{3}$ and Humphreys et al. ${ }^{4}$ The experimental setup is then described, leading to the results section. This is followed by preliminary deconvolution attempts.

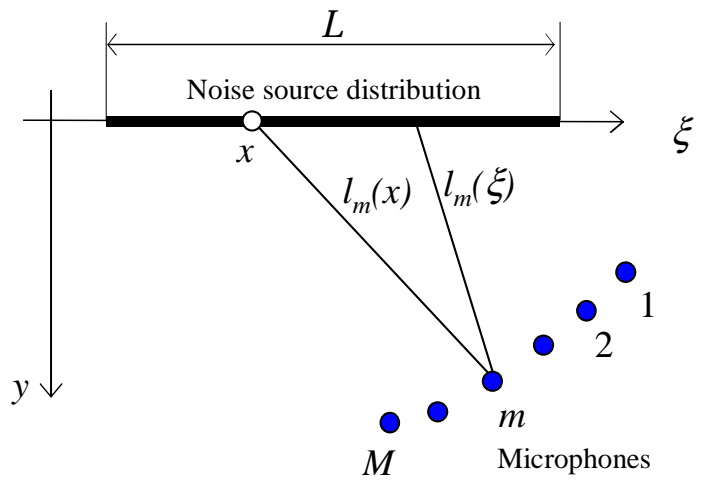

Fig.1 Linear distribution of noise sources and microphone array.

\section{Beamforming Theory}

Consider a line source distribution $q(\xi, t)$ and a collection of $M$ microphones, as depicted in Fig. 1. Assuming spherical propagation in a quiescent medium with uniform speed of sound $a$, the signal received by the $m^{\text {th }}$ microphone is

$$
p_{m}(t)=\int_{\mathcal{L}} \frac{1}{\ell_{m}(\xi)} q\left(\xi, t-\frac{\ell_{m}(\xi)}{a}\right) d \xi
$$

Integration is carried over the region of interest $\mathcal{L}$ where sound sources are expected. To focus the microphones at point $x$ along the source distribution, each signal is delayed by

$$
\tau_{m}=\frac{\ell_{m}(x)}{a}
$$

and then all the signals are summed. It is customary to weigh each microphone signal by a weighting factor $w_{m}$ before summation. Using this delay-and-sum method, the array output in time domain is

$$
s(t)=\sum_{m=1}^{M} w_{m} p_{m}\left(t+\tau_{m}\right)
$$

The weights $w_{m}$ can be constants or functions of $x$ and frequency $\omega$. In terms of the source distribution (Eq. 1 ), the array output is

$$
s(x, t)=\sum_{m=1}^{M} \int_{\mathcal{L}} \frac{w_{m}}{\ell_{m}(\xi)} q\left(\xi, t+\frac{\ell_{m}(x)-\ell_{m}(\xi)}{a}\right) d \xi
$$

To obtain the array output in frequency domain, we take the Fourier transform of Eq. 3:

$$
S(x, \omega)=\int_{-\infty}^{\infty} \sum_{m=1}^{M} w_{m} p_{m}\left(t+\tau_{m}\right) e^{-i \omega t} d t
$$

Equivalently, this can be written in terms of the source distribution (Eq. 4),

$$
S(\omega)=\int_{\mathcal{L}} Q(\xi, \omega)\left[\sum_{m=1}^{M} \frac{w_{m}}{\ell_{m}(\xi)} \exp \left(i \omega \frac{\ell_{m}(x)-\ell_{m}(\xi)}{a}\right)\right] d \xi
$$


where

$$
Q(\xi, \omega)=\int_{-\infty}^{\infty} q(\xi, t) e^{-i \omega t} d t
$$

Defining the array pattern as

$$
W(x, \xi, \omega)=\sum_{m=1}^{M} \frac{w_{m}}{\ell_{m}(\xi)} \exp \left(i \omega \frac{\ell_{m}(x)-\ell_{m}(\xi)}{a}\right)
$$

the Fourier transform of the array output takes the form

$$
S(x, \omega)=\int_{\mathcal{L}} Q(\xi, \omega) W(x, \xi, \omega) d \xi
$$

which is a generalized convolution of the Fourier transform of the source distribution with the array pattern. The quantities $S(x, \omega)$ and $Q(\xi, \omega)$ are random variables in frequency and may not even exist in a strict mathematical sense. To obtain a deterministic measure of the array output, we compute the array spectrum

$$
\Phi(x, \omega)=\left\langle S(x, \omega) S^{*}(x, \omega)>\right.
$$

The operation $<>$ denotes the time average and, in the case of Fourier transforms, has the specific meaning

$$
<A(\omega) B^{*}(\omega)>\equiv \lim _{T \rightarrow \infty} \frac{1}{2 T} \int_{-T}^{T} A(t) e^{-i \omega t} d t \int_{-T}^{T} B^{*}\left(t^{\prime}\right) e^{i \omega t^{\prime}} d t^{\prime}
$$

Equation 11 is applicable to auto spectra $(B=A)$ and cross spectra $(B \neq A)$. Substituting Eq. 9 in Eq. 10,

$$
\Phi(x, \omega)=\int_{\mathcal{L}} \int_{\mathcal{L}}<Q(\xi, \omega) Q^{*}(\zeta, \omega)>W(x, \xi, \omega) W^{*}(x, \zeta, \omega) d \xi d \zeta
$$

This is the general relation for the array power spectrum in terms of the cross spectral density of the source distribution. If we assume that the sources are axially incoherent,

$$
<Q(\xi, \omega) Q^{*}(\zeta, \omega)>=\Psi(x, \omega) \delta(\xi-\zeta)
$$

the double integral of Eq. 12 collapses to

$$
\Phi(x, \omega)=\int_{\mathcal{L}} \Psi(\xi, \omega) W(x, \xi, \omega) W^{*}(x, \xi, \omega) d \xi
$$

Defining the point spread function as

$$
V(x, \xi, \omega) \equiv W(x, \xi, \omega) W^{*}(x, \xi, \omega)=|W(x, \xi, \omega)|^{2}
$$

Eq. 14 becomes

$$
\Phi(x, \omega)=\int_{\mathcal{L}} V(x, \xi, \omega) \Psi(\xi, \omega) d \xi
$$

The array power spectrum is thus a generalized convolution of the incoherent source distribution $\Psi(\xi, \omega)$ with the point spread function $V(x, \xi, \omega)$. The problem of inverting this relation (deconvolution) to extract $\Psi(\xi, \omega)$ will be discussed in Section V.

Using the definition of the array pattern, Eq. 8, the point spread function becomes

$$
V(x, \xi, \omega)=\sum_{m=1}^{M} \sum_{n=1}^{M} \frac{w_{m}(x, \omega) w_{n}(x, \omega)}{\ell_{m}(\xi) \ell_{n}(\xi)} \exp \left[\frac{i \omega}{a}\left(\ell_{m}(x)-\ell_{n}(x)-\ell_{m}(\xi)+\ell_{n}(\xi)\right)\right]
$$

where the possible dependence of the weights on beam focus location $x$ and frequency $\omega$ is stated explicitly. The summed quantities are components of a Hermitian matrix, therefore only their real parts survive:

$$
V(x, \xi, \omega)=\sum_{m=1}^{M} \sum_{n=1}^{M} \frac{w_{m}(x, \omega) w_{n}(x, \omega)}{\ell_{m}(\xi) \ell_{n}(\xi)} \cos \left[\frac{\omega}{a}\left(\ell_{m}(x)-\ell_{n}(x)-\ell_{m}(\xi)+\ell_{n}(\xi)\right)\right]
$$


The selection of microphone weights $w_{m}(x, \omega)$, also known as "shading", has direct impact on the array power spectrum. It is customary to set

$$
w_{m}=\frac{1}{\sum_{1}^{M} \bar{w}_{m}} \bar{w}_{m} \ell_{m}(x)
$$

where $\bar{w}_{m}$ are non-dimensional weights. This filters out the effect of distance of each microphone from the focal point and, as evident from Eq. 17, gives $V(x, x, \omega)=1$. A more important consideration, however, is for the array power spectrum $\Phi(x, \omega)$ to reflect the actual intensity distribution of the noise sources $\Psi(x, \omega)$. For that, the integral of $V(x, \xi, \omega)$ should be independent of the beam focus position $x$. Since $V(x, \xi, \omega)$ has a main lobe and (undesirable) sidelobes, a more specific constraint is that the area under the main lobe be preserved. This area is approximated here as the beam width $b$ times the height of the point spread function:

$$
I_{\mathrm{ML}}=\int_{\text {main lobe }} V(x, \xi, \omega) d \xi \sim b(\omega) V(x, x, \omega)
$$

Note that the frequency dependence of $V(x, x, \omega)$ comes only from the weights. For the present array geometry, the shading of Eq. 18 results in very large variation of $I_{\mathrm{ML}}$ over the region of interest, which causes an unphysical representation of the noise source distribution. On the other hand, setting $w_{m} \sim \bar{w}_{m} y_{m}$ satisfies the integral constraint to within $10 \%$. To account for the fact that $b \sim \lambda \sim 1 / \omega$, we include the frequency dependence $w_{m} \sim \sqrt{\omega}$ or $w_{m} \sim \sqrt{S r}$. The resulting form for the weights is

$$
w_{m}=\frac{R}{\sum_{1}^{M} \bar{w}_{m}} \bar{w}_{m} \frac{y_{m}}{y_{a}} \sqrt{S r}
$$

This shading, which depends only on frequency and vertical positions of the microphones, was used in the computation of the array power spectrum from the experimental data. An added advantage is that array observation polar angle, defined as the weighted average of the microphone polar angles

$$
\theta_{\mathrm{wa}}=\frac{\sum_{1}^{M} w_{m} \theta_{m}}{\sum_{1}^{M} w_{m}}
$$

is independent of beam focus point. Examples of the axial variation of beam width and $I_{\mathrm{ML}}$ will be shown in Section III.B.

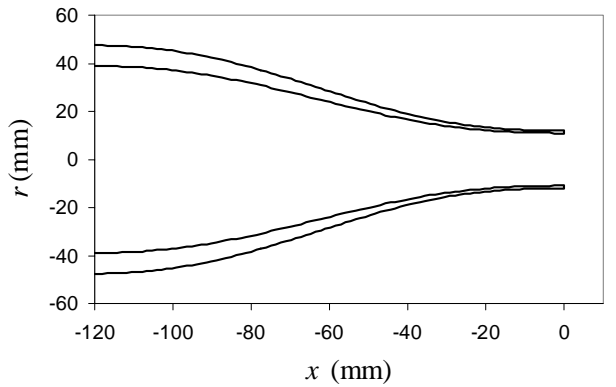

Fig. 2 Nozzle coordinates.

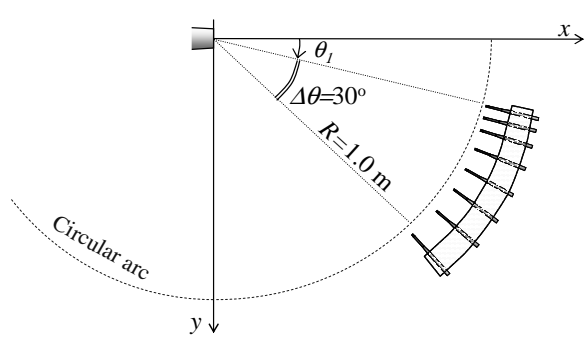

Fig. 3 Geometry of microphone phased array.

\section{Experimental Setup}

\section{A. Flow facility}

Experiments were conducted in UCI's Jet Aeroacoustics Facility, described in earlier publications. ${ }^{14}$ The facility was operated in single-stream mode with pure air, at ambient reservoir temperature, supplied to the 
convergent round nozzle shown in Fig. 2. The nozzle exit diameter was $21.8 \mathrm{~mm}$. The nozzle pressure ratio was 1.69 resulting in exit Mach number $M_{j}=0.9$ and exit velocity $U_{j}=287 \mathrm{~m} / \mathrm{s}$. The jet Reynolds number was $5 \times 10^{5}$.

\section{B. Microphone Phased Array}

The microphone phased array consists of eight 3.2-mm condenser microphones (Brüel \& Kjær Model 4138) arranged on a circular arc centered at the vicinity of the nozzle exit. Figure 3 shows the array geometry. The polar aperture of the array for this experiment was $30^{\circ}$ and the array radius was $1 \mathrm{~m}$. The angular spacing of the microphones was logarithmic, starting from $2^{\circ}$ for microphones 1 and 2 and ending with $10^{\circ}$ for microphones 7 and 8 . Uneven microphone spacing was used to mitigate the effects of spatial aliasing. The entire array structure is rotated around its center to place the array at the desired observation angle. Positioning of the array is done remotely using a stepper motor. An electronic inclinometer displays the position of first microphone. The distances between the centers of the microphone grids were measured with accuracy of $0.1 \mathrm{~mm}$ using a digital caliper. A geometric calibration procedure provided the position of each mirophone relative to the nozzle exit with accuracy of $2 \mathrm{~mm}$.

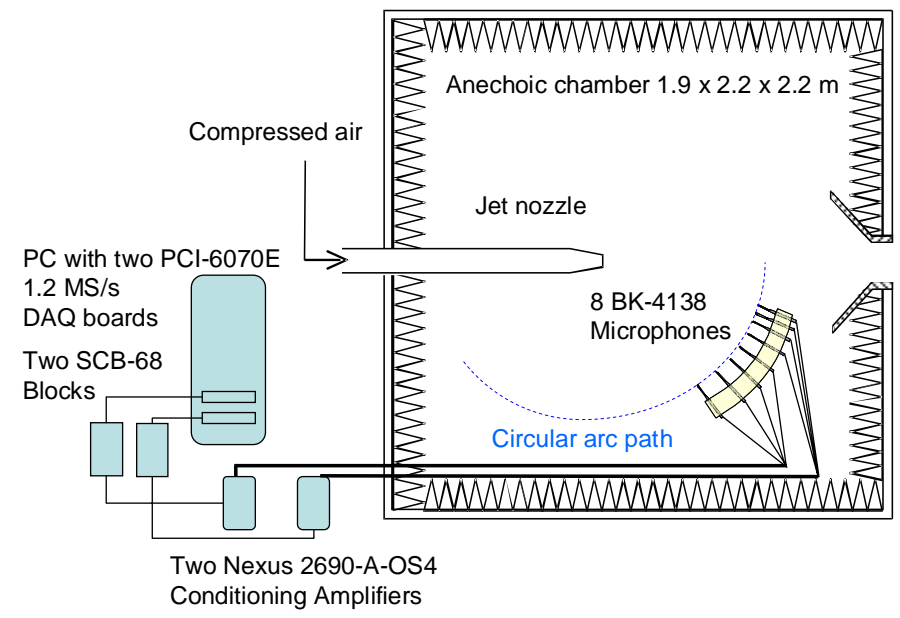

Fig.4 Primary components of microphone array system.

The arrangement of the microphones inside the anechoic chamber, and the principal electronic components, are shown in Fig. 4. The microphones were connected, in groups of four, to two amplifier/signal conditioners (Brüel \& Kjær Nexus 2690-A-OS4) with low-pass filter set at $300 \mathrm{~Hz}$ and high-pass filter set at $100 \mathrm{kHz}$. The four-channel output of each amplifier was sampled at $250 \mathrm{kHz}$ per channel by a multi-function data acquisition board (National Instruments PCI-6070E). Two such boards, one for each amplifier, were installed in a Pentium 4 personal computer. National Instruments LabView software was used to acquire the signals.

This paper discusses results obtained at two array observation angles, as defined by Eq. 21: $\theta_{\text {wa }}=30^{\circ}$ and $\theta_{\mathrm{wa}}=90^{\circ}$. They correspond to noise generated by large-scale and fine-scale turbulence, respectively. The placement of the microphones for each observation angle is plotted in Fig. 5. Table 1 provides the microphone angles and non-dimensional weights for each array position. The non-dimensional weights were selected so that the beamwidth in the middle of the region of interest (around $x / D=5$ ) was roughly the same for the two array positions. Figure 6 plots the axial distributions of the beamwidth and the integral under the main lobe of the point spread function (Eq. 19) for the two array positions. The slow variation of $I_{\mathrm{ML}}$ with $x$ is noted. If one selected the shading of Eq. 18, the variation of $I_{\mathrm{ML}}$ for $\theta_{\mathrm{wa}}=30^{\circ}$ would match the variation of the beam width at the same angle, resulting in a highly distorted representation of the noise sources. 
Table 1 Microphone Array Parameters

\begin{tabular}{|c|c|c|c|}
\hline \multicolumn{2}{|c|}{$\theta_{\mathrm{wa}}=30^{\circ}$} & \multicolumn{2}{|c|}{$\theta_{\mathrm{wa}}=90^{\circ}$} \\
\hline$\theta$ & $\bar{w}$ & $\theta$ & $\bar{w}$ \\
\hline 17.1 & 1.00 & 76.8 & 0.56 \\
\hline 19.0 & 1.00 & 78.6 & 0.67 \\
\hline 21.3 & 1.00 & 80.8 & 0.80 \\
\hline 23.9 & 1.00 & 83.3 & 0.92 \\
\hline 27.1 & 1.00 & 86.4 & 1.00 \\
\hline 31.3 & 1.00 & 90.4 & 0.95 \\
\hline 37.0 & 1.00 & 95.0 & 0.57 \\
\hline 45.8 & 1.00 & 105.5 & 0.17 \\
\hline
\end{tabular}

a)

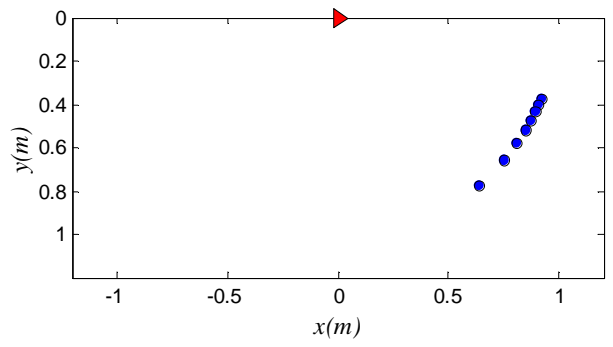

b)

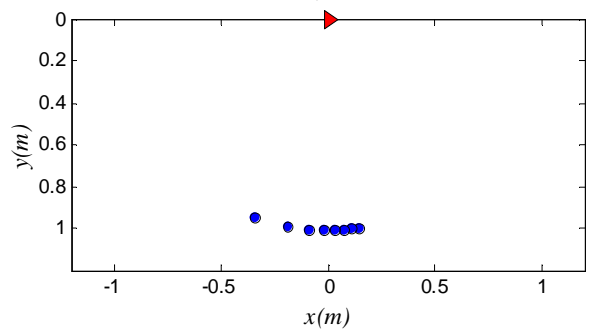

Fig.5 Two positions of array: a) $\theta_{\mathrm{wa}}=30^{\circ}$; b) $\theta_{\mathrm{wa}}=90^{\circ}$. Triangles indicate nozzle exit.
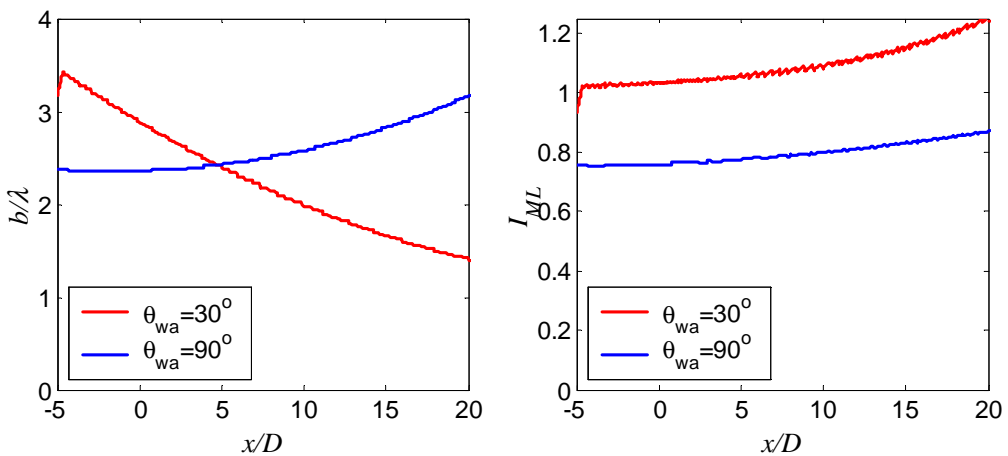

Fig.6 Axial variations of beamwidth, normalized by wavelength, and $I_{\mathrm{ML}}$ for the two positions of the array.

\section{Data Processing}

Insertion of Eq. 5 into the definition of the array power spectrum, Eq. 10, gives

$$
\Phi(x, \omega)=\sum_{m=1}^{M} \sum_{n=1}^{M} w_{m} w_{n} e^{i \omega\left(\tau_{m}-\tau_{n}\right)}<P_{m}(\omega) P_{n}^{*}(\omega)>
$$

where

$$
P_{m}(\omega)=\int_{-\infty}^{\infty} p_{m}(t) e^{-i \omega t} d t
$$

Defining the cross-spectrum matrix as

$$
G_{m n} \equiv<P_{m}(\omega) P_{n}^{*}(\omega)>
$$


we have

$$
\Phi(x, \omega)=\sum_{m=1}^{M} \sum_{n=1}^{M} w_{m} w_{n} e^{i \omega\left(\tau_{m}-\tau_{n}\right)} G_{m n}
$$

or

$$
\Phi(x, \omega)=\epsilon \mathbf{G} \epsilon^{T}
$$

where

$$
\epsilon_{m}(x, \omega)=w_{m}(x, \omega) e^{i \omega \tau_{m}(x)}
$$

is the weighted steering vector and superscript $T$ denotes its complex transpose. Eq. 25 formed the basis for the computation of the array power spectrum from the microphone pressure traces. The weights were selected according to Eq. 20 with $\bar{w}_{m}$ listed in Table 1.

The computation of the cross spectrum matrix, Eq. 24, involved the following steps. Each microphone signal consisted of $N_{s}=2^{18}=262144$ samples acquired at a sampling rate $F_{s}=250 \mathrm{kHz}$. The maximum resolvable (Nyquist) frequency was $F_{s} / 2=125 \mathrm{kHz}$, although the high-pass filter was set a little lower at $f=100 \mathrm{kHz}$. The size of the Fast Fourier Transform was $N_{\mathrm{FFT}}=2024$ yielding a frequency resolution of $122 \mathrm{~Hz}$. Each signal was divided into $K=64$ blocks of 4096 samples each, and the data within each block was windowed using a Hamming window. The cross-spectrum matrix $G_{m n}^{k}$ for block $k$ was computed using Fortran routines for autospectra and crossspectra. The total cross-spectrum matrix was obtained from

$$
G_{m n}(f)=\frac{1}{K W_{h}} \sum_{k=1}^{K} G_{m n}^{k}(f)
$$

where $W_{h}$ is a weighting constant for the Hamming window. Since the cross-spectrum matrix is Hermitian, only the diagonal and upper-triangle elements were computed; the lower-triangle elements were calculated as complex conjugates of the upper-triangle elements.

To present the array power spectrum in the form of lossless sound pressure level spectrum (units of decibels), the following procedure was used:

$$
\Phi_{\mathrm{SPL}}(x, f)=10 \log _{10}[\Phi(x, f)]+93.98-C_{\mathrm{fr}}(f)-C_{\mathrm{ff}}(f)+\alpha(f) \ell_{a}(x)
$$

The constant 93.98 comes from the normalization of the pressure by the reference pressure of $20 \mu \mathrm{Pa}$, that is, $-20 \log _{10}\left(20 \times 10^{-6}\right)=93.98$. $C_{\mathrm{fr}}$ and $C_{\mathrm{ff}}$ are the corrections for the actuator response and free-field response, respectively; they are based on data provided by the manufacturer of the microphone and are practically the same for all the microphones. $\alpha$ is the atmospheric absorption coefficient $(\mathrm{dB} / \mathrm{m})$, computed using the formulas proposed by Bass et al. ${ }^{16}$ for the measured values of relative humidity and temperature of the ambient air. The absorption correction is based on the average distance $\ell_{a}(x)$ of the microphones from the focus point.

As in many acoustic experiments, microphone spectra contain wiggles which are unrelated to jet noise physics and are often caused by reflections from surfaces near the microphones, such as microphone mounts. Even though great care was taken to minimize such surfaces, the spectra in our experiments contain a small level of wiggles. The wiggles can be removed by smoothing the spectrum using filters that preserve its basic shape. This improves dramatically the presentation of the results. A Savitzky-Golay low-pass filter ${ }^{15}$ was used for this purpose. It was applied on either the cross spectrum matrix or on the array power spectrum, with same results. The filter size was variable and ranged from 3 at low frequencies to 100 at high frequencies (each spectrum contains 1024 points). An example of the smoothing is shown in Fig. 7. It is seen that the smoothing process preserves the fundamental shape of the spectrum. This type of filterng assumes that the spectrum is inherently smooth, which is the case for the subsonic Mach number of this experiment. For supersonic flows, one needs to account for the possibility of resonant tones, in which case smoothing must be done in a manner that preserves those tones. 


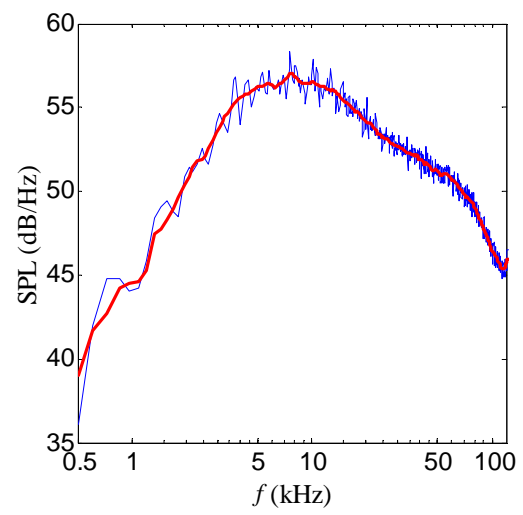

Fig.7 Example of smoothing of array power spectrum.

\section{Direct Results}

We discuss results arising directly from the beamforming procedure of Section III.C, that is, prior to deconvolution attempts. Figure 8 presents isocontours of $\Phi_{\mathrm{SPL}}(x, S r)$ for the two array observation angles. For $\theta_{\text {wa }}=30^{\circ}$ (the direction influenced by noise from large-scale structures) we note distributions that appear fairly elongated axially, particularly at the low frequencies. The maximum level is located at $x / D=6$ and $S r=0.2$. The axial location of the maximum level is close to the end of the potential core, as determined by mean velocity surveys of this flow (see for example Fig. 12 of Ref. 16). It is clear from the isocontours that, as the frequency increases, the peak noise source level moves closer to the nozzle exit. The map of the array power spectrum for $\theta_{\text {wa }}=90^{\circ}$ (the direction influenced by noise from fine-scale structures) is quite different. The range of levels is smaller reflecting the flatter spectra in that direction. Even though at first sight the distribution appears more confined axially than the distribution at $\theta_{\text {wa }}=30^{\circ}$, we will see that, in fact, this is not the case and that the different appearance of the power spectrum is due mainly to the difference in spectral shape. The axial location of the maximum level is $x / D \approx 4$, slightly upstream of the the maximum level $\theta_{\text {wa }}=30^{\circ}$, indicating that the strongest sources of fine-scale noise also reside near the end of the potential core. The Strouhal number associated with the maximum level at $\theta_{\mathrm{wa}}=90^{\circ}$ is around 0.5. The trend of the peak noise source moving towards the nozzle exit as frequency increases is evident for this observation angle too. The effects of the sidelobes are evident for both observation angles.

It is important to recall that the plots of Fig. 8 represent a convolution between the noise source distribution and the point spread function. One may question whether the differences noted between small and large array angles could be an artifact of convolution. In this vein, it is noted that the weights were selected such that the array beam width was essentially the same at $\theta_{\text {wa }}=30^{\circ}$ and $\theta_{\text {wa }}=90^{\circ}$ near $x / D=5$ (Fig. 6). Therefore, the convolution should not have played a material role in the distinct appearances $\Phi_{\mathrm{SPL}}(x, S r)$ for the two observation angles.

We proceed with extracting more information from Fig. 8. Figure 9 shows the axial distributions of $\Phi_{\mathrm{SPL}}$ for various Strouhal numbers. Even though the absolute levels are different for the two array angles, the shapes of the distributions are comparable. The difference in absolute levels is caused by the different frequency spectra at the two angles, as will be shown shortly. Figure 10 plots the location of the peak noise source on the $x / D-S r$ plane. The peak noise source location maps for the two array angles are roughly similar, with minor differences discussed below. Broadly speaking, as the Strouhal number increases from 0.1 to 10, the location of the peak noise moves upstream from $x / D \approx 7$ to $x / D=0$. This trend is similar to that found in cold subsonic jets by Narayanan et $\mathrm{al}^{5}$ and by Lee and Bridges. ${ }^{6}$ Near $S r=0.3$ both source location plots do a small "zig-zag" that is also noticeable in some of the noise source location plots of Lee and Bridges. The significance of this pattern is not clear. One difference between the two angles is that, at $S r \sim 0.3$, the peak noise for $\theta_{\mathrm{wa}}=30^{\circ}$ occurs roughly one to two jet diameters downstream of the peak noise for $\theta_{\mathrm{wa}}=30^{\circ}$. The spectra along the loci of peak noise for $\theta_{\mathrm{wa}}=30^{\circ}$ and $90^{\circ}$, plotted in Fig. 11, are representative of the 
single-microphone spectra for noise generated from large-scale and fine-scale turbulence, respectively.

a)

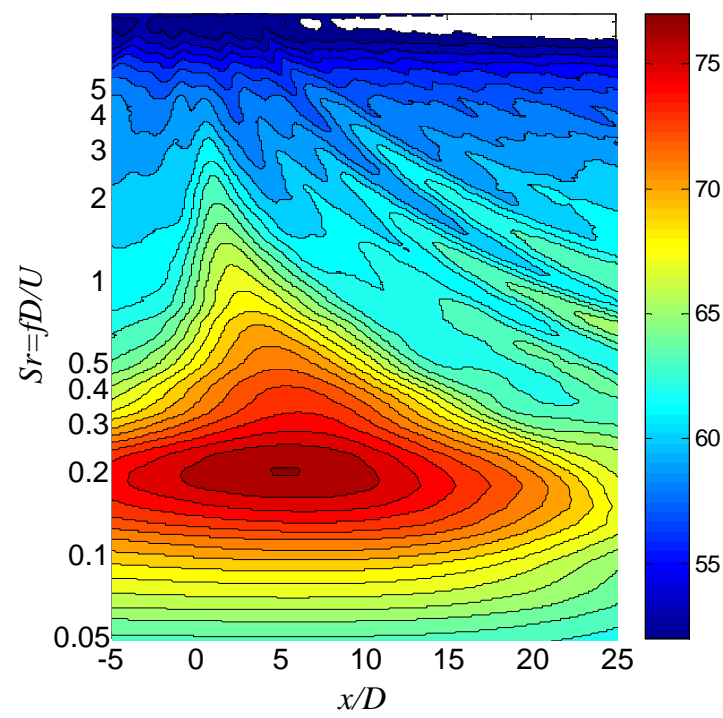

b)

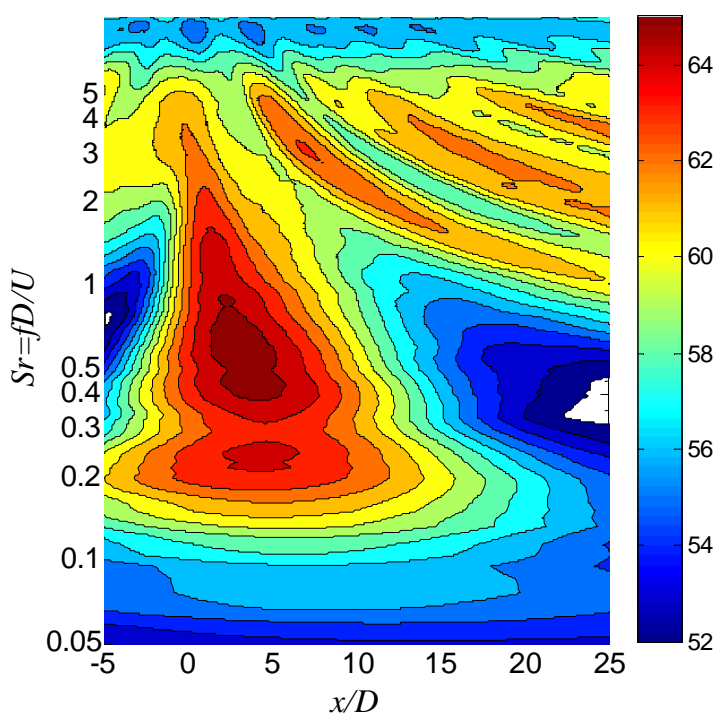

Fig.8 Isocontours of $\Phi_{\mathrm{SPL}}$. a) $\theta_{\mathrm{wa}}=30^{\circ}$; b) $\theta_{\mathrm{wa}}=90^{\circ}$.

a)

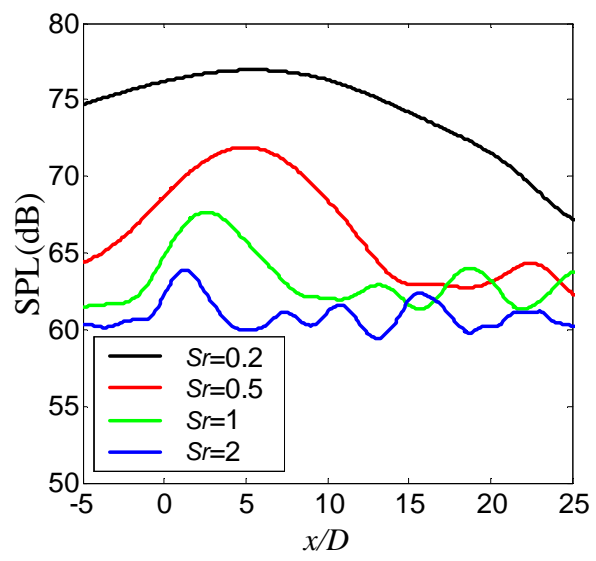

b)

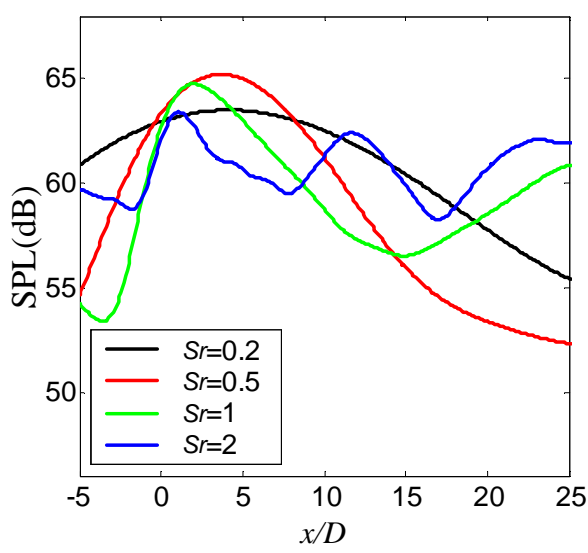

Fig.9 Axial distribution of $\Phi_{\mathrm{SPL}}$ for several Strouhal numbers. a) $\theta_{\mathrm{wa}}=30^{\circ}$; b) $\theta_{\mathrm{wa}}=90^{\circ}$.

\section{Deconvolved Results}

As discussed in Section II, the array power spectrum is a generalized convolution between the incoherent source distribution and the point spread function, as described by Eq. 16:

$$
\Phi(x, \omega)=\int_{\mathcal{L}} V(x, \xi, \omega) \Psi(\xi, \omega) d \xi
$$

In other words, one obtains a blurred and distorted image of the sources, where the blurring/distortion depends on the shape of the point spread function. An intriguing proposition is to invert Eq. 16 to get a more accurate estimate of the noise source distribution. In general this is a difficult task that may not have a unique answer. This section presents preliminary attempts to invert Eq. 16 and obtain the "true" noise source distribution. 


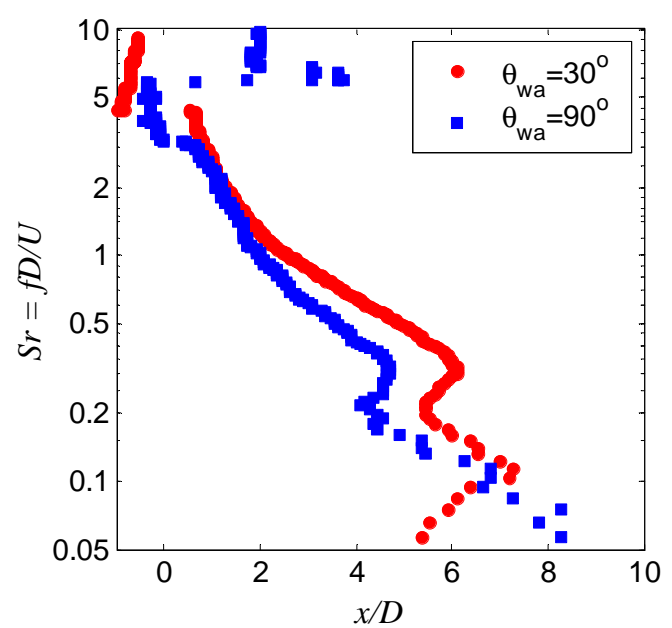

Fig.10 Location of peak noise on $x / D-$ Sr diagram.

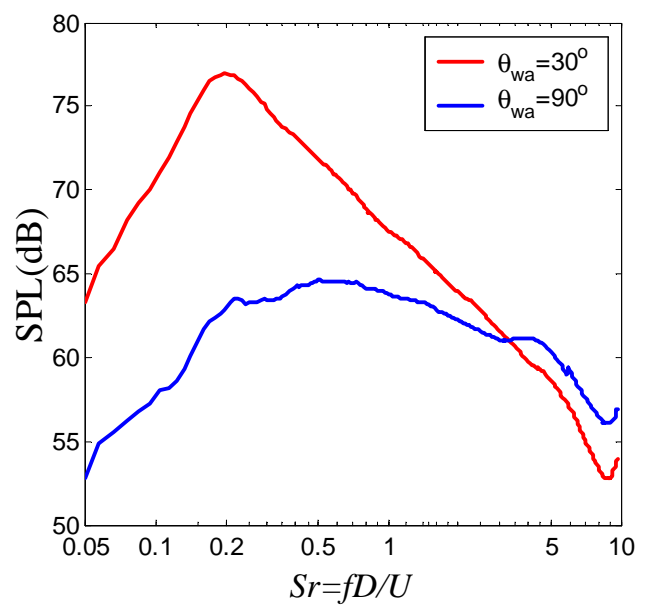

Fig.11 Spectra along the loci of peak noise of Fig. 10.

\section{A. Inversion Algorithm}

For a given frequency, the integral of Eq. 16 can be expressed as a summation over a finite number of sources $N$. Applying the following discretizations,

$$
\begin{gathered}
\Phi(x, \omega) \rightarrow \Phi_{i} \\
V(x, \xi, \omega) \rightarrow V_{k i} \\
\Psi(\xi, \omega) \Delta \xi \rightarrow \Psi_{k}
\end{gathered}
$$

Eq. 16 becomes the linear system of equations

$$
\Phi_{i}=\sum_{k=1}^{N} V_{i k} \Psi_{k}
$$

or

$$
\Phi=\mathrm{V} \Psi
$$

The discrete form of the point spread function, Eq. 17, is

$$
V_{i k}=\sum_{m=1}^{M} \sum_{n=1}^{M} \frac{w_{m, i} w_{n, k}}{\ell_{m, k} \ell_{n, k}} \cos \left[\frac{\omega}{a}\left(\ell_{m, i}-\ell_{n, i}-\ell_{m, k}+\ell_{n, k}\right)\right]
$$

where $\ell_{m, k}$ is the distance of microphone $m$ from axial location $k, \ell_{m, i}$ is the distance of microphone $m$ from focus point $i$, etc.

Recent approaches to deconvolving the array output include the DAMAS algorithm of Brooks and Humphreys ${ }^{9}$ and the DAMAS2 and DAMAS3 algorithms of Dougherty. ${ }^{10}$ Dougherty's algorithms assume that the point spread function is - or can be rendered - in convolutional form, i.e., $V(x, \xi, \omega)=V(x-\xi, \omega)$, which is not the case for the present array. The DAMAS algorithm is basically a Gauss-Seidel (GS) iterative method, ${ }^{18}$ with non-negative constraint imposed at each iteration step. The performance of GS can depend on the ordering of the equations. The DAMAS approach was tried and, while the results seemed reasonable, their dependence on the order of the equations raised concern. For example, the location of peak noise changed by as much as three jet diameters depending on whether the rows of the linear system of Eq. 31 were ordered forward or backward in $x$. 
Another method tested was the Richardson-Lucy (RL) iteration algorithm ${ }^{19,20}$ which has found wide application in image restoration. The advantage of RL over GS is that its output is inherently non-negative and does not depend on the sequence of the equations. Several tests indicated that the output was consistently smoother and more robust than that of the GS method. For these reasons, the RL method was selected for the deconvolution. The iteration algorithm is

$$
\begin{aligned}
\Psi_{k}^{(j)} & =\Psi_{k}^{(j-1)} \frac{1}{\sum_{i=1}^{N} V_{i k}} \sum_{i=1}^{N} \frac{V_{i k} \Phi_{i}}{\tilde{\Phi}_{i}} \\
\tilde{\Phi}_{i} & =\sum_{k=1}^{N} V_{i k} \Psi_{k}^{(j-1)}
\end{aligned}
$$

The spatial extent of the region investigated and the resolution of grid points are critical parameters for the success of the deconvolution scheme. The guidance of Brooks and Humphreys ${ }^{9}$ was followed in setting these parameters. As shown in Fig. 12, the investigation domain consists of two overlapping regions: the scan region over which Eq. 33 is applied and the region of interest that includes the relevant noise sources. The scan region is larger than or equal to the region of interest. The length of the scan region $\Lambda$ should be greater than the beam width and was selected to be $\Lambda=2 b$. For the present array it was determined that $b \approx 2 \lambda$, therefore $\Lambda=4 \lambda$. The region of interest was chosen as $-5 D<x<25 D$, so its length was $\mathcal{L}=30 D$. The scan region was arranged symmetrically over the region of interest, as shown in Fig. 12. The spatial resolution should be a fraction of the wavelength and was set at $\Delta \xi=0.25 \lambda$. However, at low frequencies where the wavelength is very large, the resolution was set at $\Delta \xi=0.01 \mathcal{L}$. The combined scheme for the resolution was

$$
\Delta \xi=\min (0.01 \mathcal{L}, 0.25 \lambda)
$$

Typically, the method converged to $r^{(j)}<0.05$ in 50 iterations, with slow improvement thereafter. The resolution of point sources, discussed below, kept improving with $j$ increasing up to 200. For this reason the number of iterations was set at 200 .

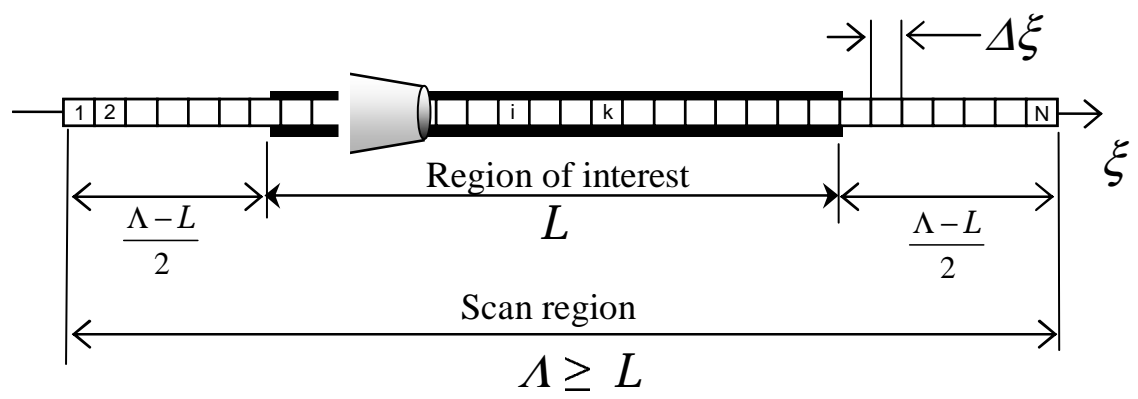

Fig.12 Scan region and region of interest for deconvolution algorithm.

\section{B. Point Sources}

We evaluate the performance of the deconvolution scheme for synthetic and physical point sources. The synthetic source is effectively a Dirac delta function placed at $\xi=x_{s}$, which renders the left hand side of Eq. 16 equal to $V\left(x, x_{s}, \omega\right)$. Equation 33 is thus asked to invert the relation

$$
V\left(x, x_{s}, \omega\right)=\int_{\mathcal{L}} V(x, \xi, \omega) \Psi(\xi, \omega) d \xi
$$

for which the answer should be

$$
\Psi=\delta\left(\xi-x_{s}\right)
$$


The performance of the deconvolution process for a synthetic point source is shown in Fig. 13, which plots the left- and right- hand sides of Eq. 31, and the resulting source distribution, for various frequencies and for the two array polar angles. Inversion of Eq. 34 yields a source distribution that is much narrower than the array power spectrum and resembles a delta function at high frequencies. At low frequencies, the effective beam width is reduced by factor of about 5, this factor increasing with rising frequency. The spatial resolution becomes finer than one jet diameter for $S r>0.4$. For $S r<0.1$, the effective beam width is still too large compared to the jet diameter. At low frequencies, the effective beam width at $\theta_{\text {wa }}=30^{\circ}$ is slightly larger than that at $\theta_{\mathrm{wa}}=90^{\circ}$.
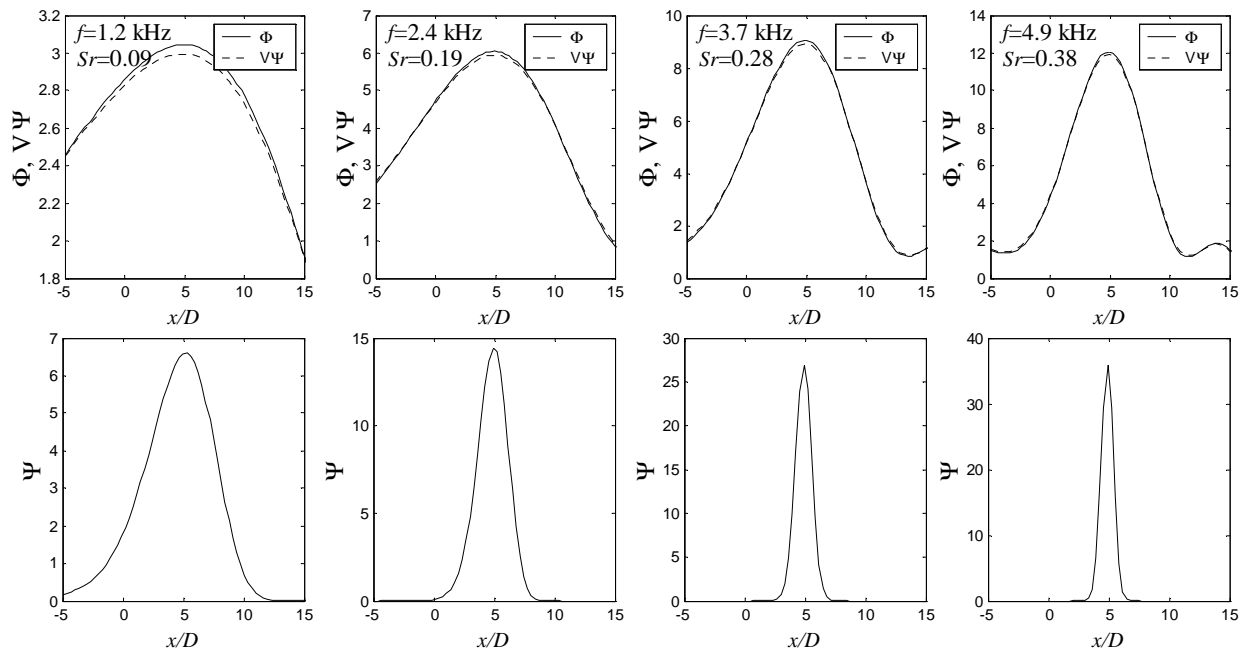

a)
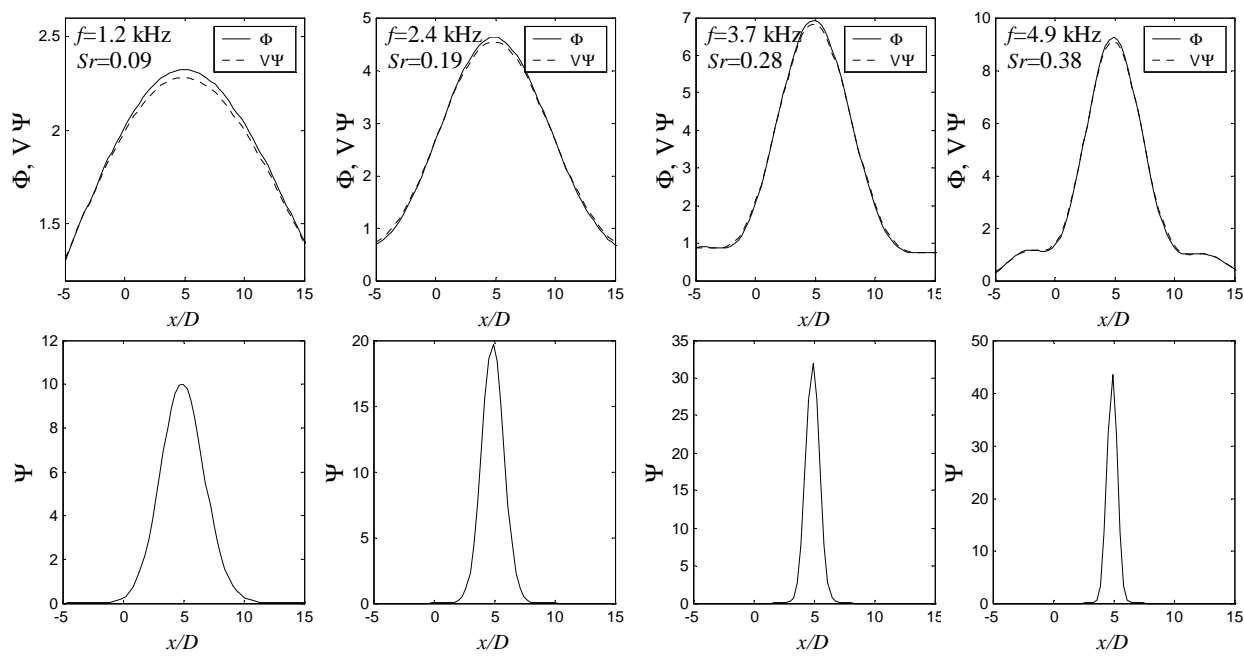

b)

Fig.13 Performance of deconvolution for synthetic point source situated at $x / D=5$. a) $\theta_{\mathrm{wa}}=30^{\circ}$; b) $\theta_{\text {wa }}=90^{\circ}$.

The physical point source was produced by a speaker excited by a broadband signal. Sound from the speaker was funneled down a tube with exit diameter of $5 \mathrm{~mm}$. The axis of the tube was perpendicular to the plane of the array arc and the outlet of the tube was placed on the jet axis a small distance from the position where the nozzle terminates when installed. The nozzle was removed and the piping that supplies the nozzle was covered with anechoic foam to prevent reflections. Figure 14 plots the point spread function, left- and right-hand sides of Eq. 31, and the resulting source distribution for $f=10 \mathrm{kHz}$ and the two array polar angles. The good match between the array power spectrum and the point spread function indicates that this arrangement produced a well-defined point source. The deconvolved source distribution is very close to 
a delta function.

a)
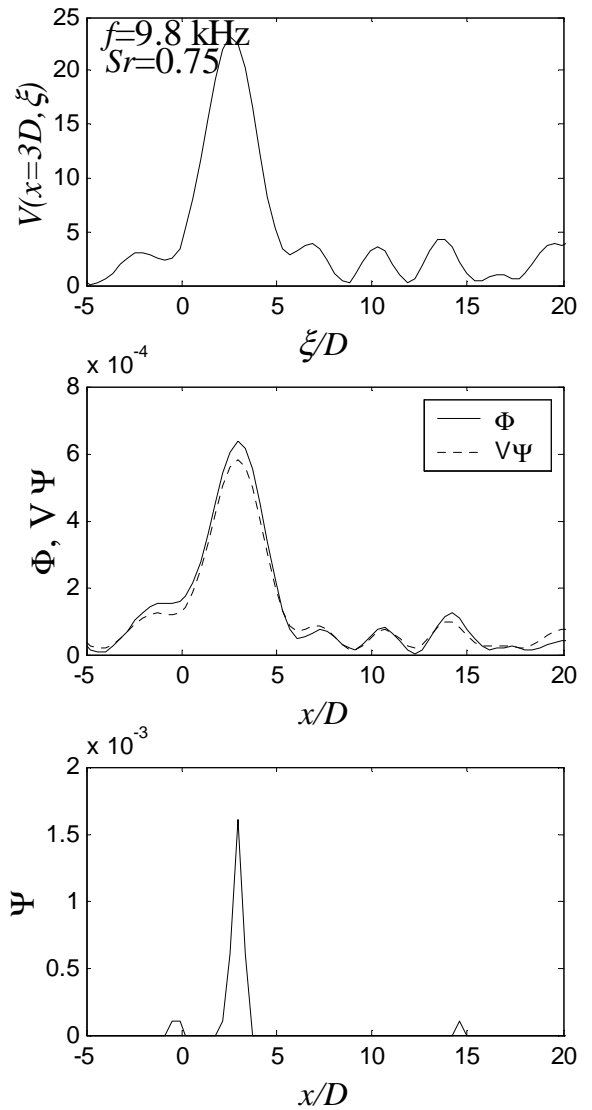

b)
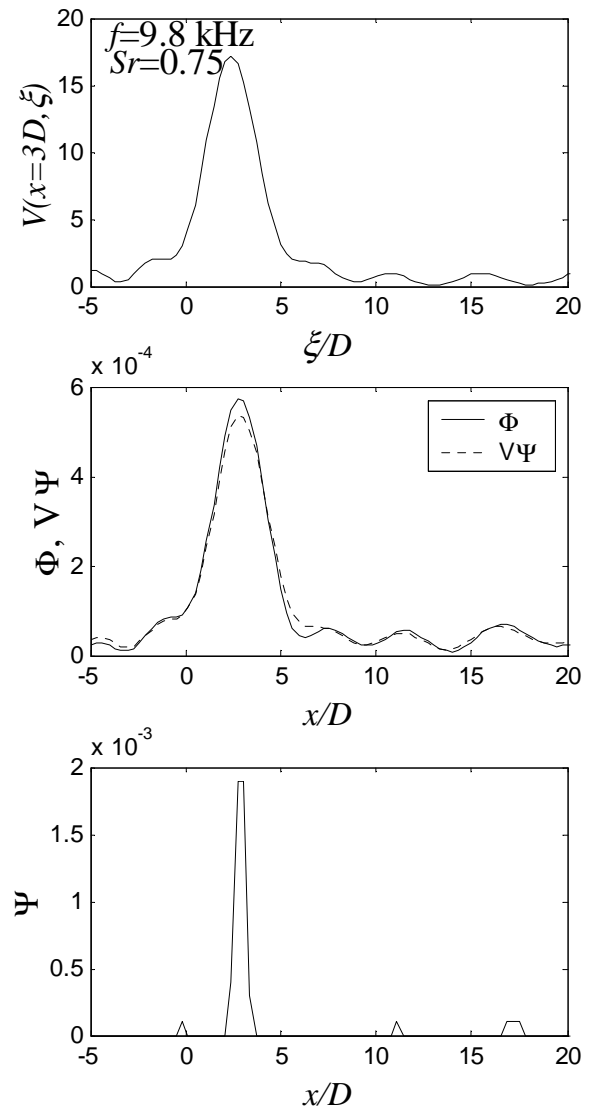

Fig.14 Performance of deconvolution for physical point source situated at $x / D=3$. a) $\theta_{\text {wa }}=30^{\circ}$; b) $\theta_{\text {wa }}=90^{\circ}$.

\section{Jet Noise}

Isocontours of the deconvolved sound pressure level spectrum are shown in Fig. 17 for $\theta_{\text {wa }}=30^{\circ}$ and $90^{\circ}$. White regions indicate levels below the range of the color bars. Comparing with the direct results of Fig. 8 , the noise source region is more confined, a result of the smaller effective beam width. For both angles, at $S r>0.4$ there is a sharp cutoff of the noise sources at $x / D=0$, indicating that deconvolution process results in an accurate representation of the noise source distribution when the beam width becomes less than the order of one jet diameter (see Section V.B). The sidelobes are still visible but become more separable than in the direct results. Generally speaking, the differences between the two observation polar angles are similar to those noted in the direct results of Fig. 8.

Figure 16 plots the axial distribution of the deconvolved sound pressure level spectrum at three Strouhal numbers and for the two array observation angles. The appearance of sidelobes in the figures becomes more pronounced as the frequency increases but they can be easily distinguished from the main result and thus discounted. The similarity in the shape of the distributions at $\theta_{\mathrm{wa}}=30^{\circ}$ and $\theta_{\mathrm{wa}}=90^{\circ}$ is notable. The results suggest that the same axial growth-decay process governs the generation of noise from large-scale and fine-scale turbulence.

The location of peak noise on the $x / D-S r$ diagram is plotted in Fig. 17 for the two observation polar angles. The same trends noted in the direct results of Fig. 10 are observed here also. The spectra along the 
a)

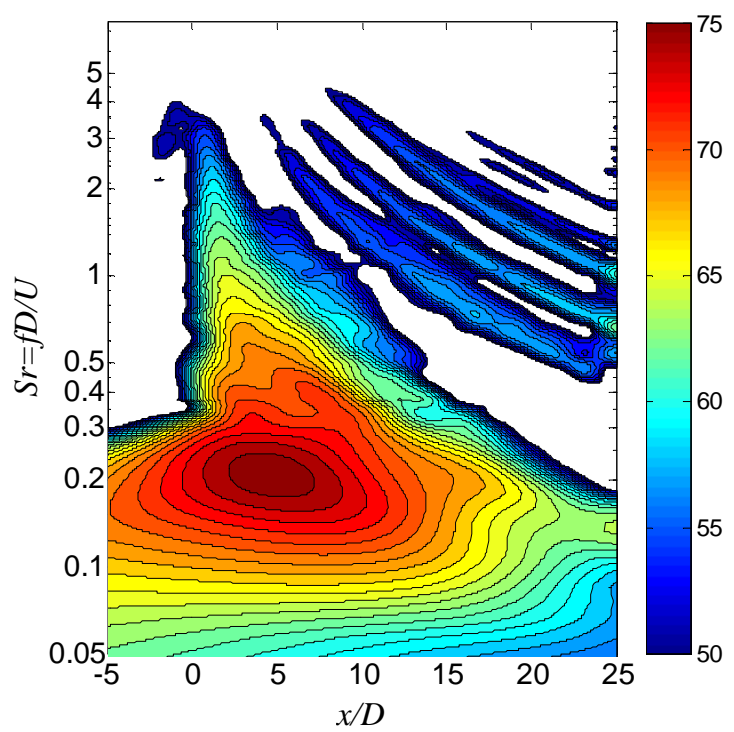

b)

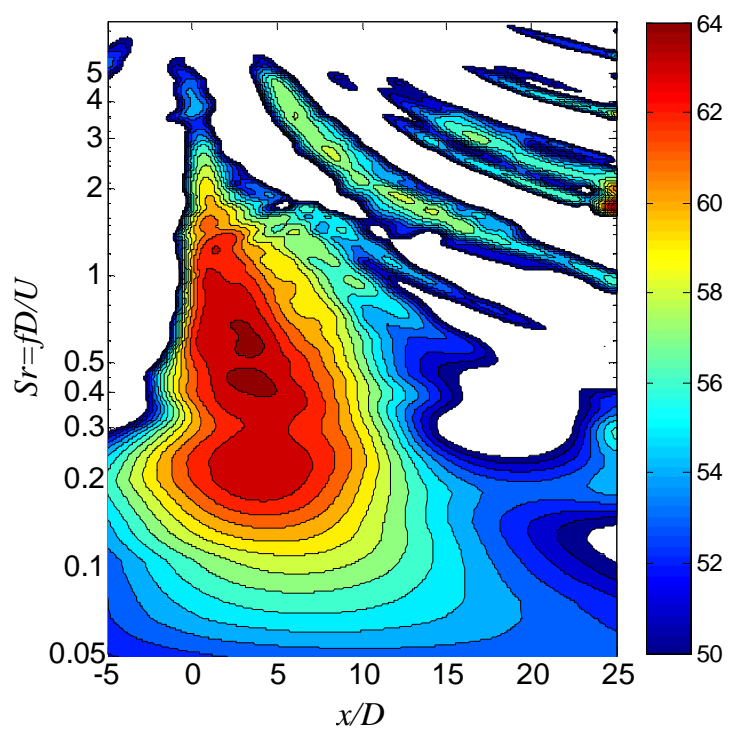

Fig.15 Isocontours of deconvolved $\Phi_{\mathrm{SPL}}$. a) $\theta_{\mathrm{wa}}=30^{\circ}$; b) $\theta_{\mathrm{wa}}=90^{\circ}$.
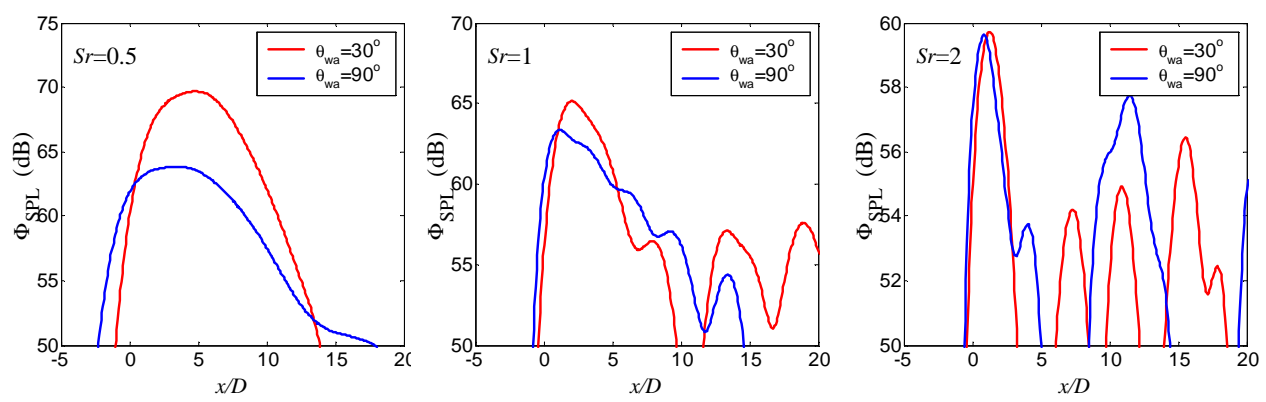

Fig.16 Axial distributions of deconvolved $\Phi_{\mathrm{SPL}}$ for $\theta_{\mathrm{wa}}=30^{\circ}$ and $\theta_{\mathrm{wa}}=90^{\circ}$ at three Strouhal numbers.

loci of peak noise, shown in Fig. 18, have the same characteristics as the corresponding spectra of the direct results (Fig. 11).

\section{Concluding Remarks}

Application of phased arrays to small-scale jet experiments is a challenging proposition. The main difficulty stems from the fact that, at frequencies pertinent to full-scale aircraft noise, the wavelength becomes of the same order as the size of the microphones. This gives rise to spatial aliasing effects and free-field corrections that require careful positioning of the microphones and processing of the data. To resolve the directionality of jet noise, a small-polar-aperture array is essential. The small aperture, which results in large beam widths, magnifies the challenge. The experiment described here dealt with these issues to obtain preliminary estimates of distributions for large-scale and fine-scale noise sources in a single-stream jet. Some guidelines are proposed for the positioning of the microphones and the selection of weighting functions so that the array power spectrum gives reasonable representations of the noise source distributions.

This study examined the distribution of noise sources in a Mach 0.9 cold jet as viewed at two array polar angles, a small angle associated with emission of noise from large -scale turbulent structures and a large angle associated with noise emission from fine-scale turbulence. Examination of the space-frequency noise source maps for each angle shows similarities and differences between the two types of noise sources. Both types of 


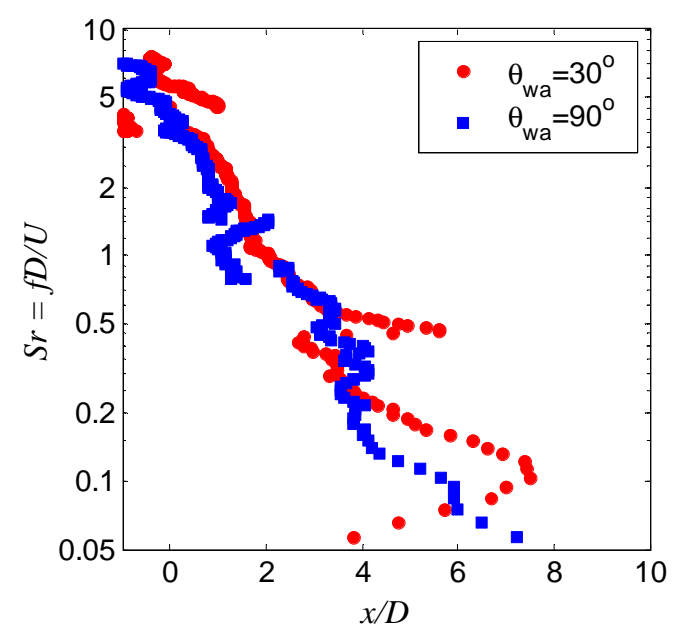

Fig.17 Location of peak noise on $x / D-$ $\mathrm{Sr}$ diagram, based on deconvolved array power spectrum.

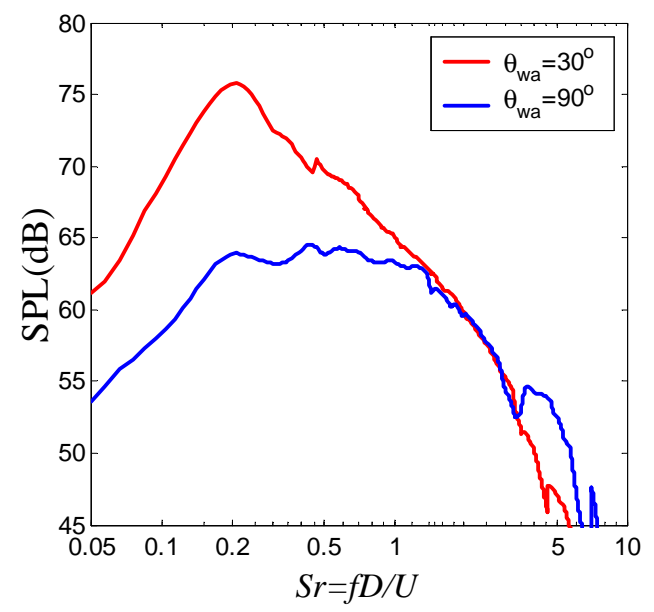

Fig.18 Spectra along the loci of peak noise of Fig. 17.

noise sources peak near $x / D=5$, which marks the end of the potential core. The Strouhal number at the peak is approximately 0.2 for the large-scale noise and 0.5 for the fine-scale noise. With increasing Strouhal number the location of the peak moves toward the nozzle exit, a trend observed for both observation polar angles. However, at low to moderate frequencies, the peak of large-scale noise sources appears to occur about 1-2 jet diameters downstream of the peak of fine-scale noise sources. The overall differences between the noise source maps are due mainly to the distinct spectral characteristics of the two types of noise sources. Along the paths of peak noise on the space-frequency diagram, the spectra at low observation angle and large observation angle are consistent with the similarity spectra ${ }^{11}$ for large-scale and fine-scale noise, respectively.

Deconvolution based on the Richardon-Lucy inversion method shows promise as a means to extract the true source distribution from the array power spectrum. Tests with synthetic and real point sources show that deconvolution reduces the effective beam width by factor of at least five. For $S r>0.4$ the spatial resolution is less then one jet diameter and the noise source maps look more realistic. The basic trends discussed above do not change significantly after deconvolution. However, the deconvolved data show more clearly the axial distributions of noise sources and the remarkable similarity of these distributions at small and large array angles.

\section{Acknowledgments}

The support by Goodrich Aerospace, Aerostructures Group, and by NASA Glenn Research Center (Grant NAG-3-2345 monitored by Dr. Khairul B. Zaman and Dr. James Bridges) is gratefully acknowledged. Dr. Robert Dougherty (Optinav Inc.) is thanked for suggesting the Richardson-Lucy deconvolution algorithm.

\section{References}

${ }^{1}$ Laufer, J., Schlinker, R., and Kaplan, R.E., "Experiments on Supersonic Jet Noise," AIAA Journal, Vol. 14, No.4, 1976, pp. 489-497.

${ }^{2}$ Fisher, M.J., Harper-Bourne, M., and Glegg, S.A.L., "Jet Engine Source Location: The Polar Correlation Technique," Journal of Sound and Vibration, Vol. 51, No.1, 1977, pp. 23-54.

${ }^{3}$ Billingsley, J. and Kinns, R., "The Acoustic Telescope," Journal of Sound and Vibration, Vol. 48, No.4, 1976, pp. 485-510. 
${ }^{4}$ Humphreys, W.M., Brooks, T.F., Hunter, W.W., and Meadows, K.R., "Design and Use of Microphone Directional Arrays for Aeroacoustic Measurements," AIAA-98-0471, Jan. 1998.

${ }^{5}$ Narayanan, S., Barber, T.J., and Polak, D.R., "High Subsonic Jet Experiments: Turbulence and Noise Generation Studies," AIAA Journal, Vol. 40, No. 3, 2002, pp. 430-437.

${ }^{6}$ Lee, S.S. and Bridges, J., "Phased-array Measurements of Single Flow Hot Jets," AIAA-2005-2842, May 2005 .

${ }^{7}$ Hileman, J., Thurow, B., and Samimy, M., "Exploring Noise Sources Using Simultaneous Acoustic Measurements in Real-Time Flow Visualizations in Jets," AIAA Journal, Vol. 40, No. 12, 2002, pp. 2382-2392.

${ }^{8}$ Venkatesh S.R., Polak D.R., and Narayanan S., "Beamforming Algorithm for Distributed Source Localization and its Application to Jet Noise," AIAA Journal, Vol. 41, No.7, 2003, pp. 1238-1246.

${ }^{9}$ Brooks, T.F., and Humphreys, W.M., "A Deconvolution Approach for the Mapping of Acoustic Sources (DAMAS) Determined from Phased Microphone Arrays," AIAA-2004-2954, May 2004.

${ }^{10}$ Dougherty, R.P., "Extensions of DAMAS and Benefits and Limitations of Deconvolution in Beamforming," AIAA-2005-2961, May 2005.

11 Tam, C.K.W., "Jet Noise: Since 1952," Theoretical and Computational Fluid Dynamics, Vol. 10, 1998, pp. 393-405.

12 Tam, C.K.W., Golebiowski, M., and Seiner, J.M., "On the Two Components of Turbulent Mixing Noise from Supersonic Jets," AIAA Paper 96-1716, May 1996.

13 Tam, C.K.W, Pastouchenko, N.N., and Schlinker, R.H., "Noise Source Distribution in Supersonic Jets," Journal of Sound and Vibration, Vol. 291, Nos.1-2, 2006 pp. 192-201.

${ }^{14}$ Papamoschou, D. and Debiasi, M., "Directional Suppression of Noise from a High-Speed Jet," $A I A A$ Journal, Vol. 39, No. 3, 2001, pp. 380-387.

15 Savitzky, A., and Golay, M.J.E., "Smoothing and Differentiation of Data by Simplified Least Squares Procedures," Analytical Chemistry Vol. 36, No.8, 1964, pp. 1627.

${ }^{16}$ Bass, H.E., Sutherland, L.C., Blackstock, D.T., and Hester, D.M., "Atmospheric Absorption of Sound: Further Developments, " Journal of the Acoustical Society of America, Vol. 97, No. 1, 1995, pp. 680-683.

${ }^{17}$ Papamoschou, D. " New Method for Jet Noise Reduction in Turbofan Engines," AIAA-2003-1059, Jan. 2003.

18 Franklin, J.N., "Matrix Theory," 1st ed., Prentice Hall, Englewood Cliffs, New Jersey, 1968, pp. 221-225.

${ }^{19}$ Richardson, W.H., "Bayesian-Based Iterative Method of Image Restoration," Journal of the Optical Society of America, Vol. 62, No. 1, 1972, pp. 55-

${ }^{20}$ Lucy, L.B., "Iterative Technique for Rectification of Observed Distribution," Astronomical Journal, Vol. 79, No. 6, 1964, pp. 745-754. 